\title{
The effectiveness and value of bempedoic acid and inclisiran for heterozygous familial hypercholesterolemia and secondary prevention of ASCVD
}

\author{
A summary from the Institute for Clinical and Economic Review's Midwest Comparative \\ Effectiveness Public Advisory Council
}

Foluso Agboola, MBBS, MPH; Grace A Lin, MD, MAS; Dhruv S Kazi, MD, MSc, MS; Avery McKenna; and Steven D Pearson, MD, MSc

Atherosclerotic cardiovascular disease (ASCVD) encompasses a set of chronic conditions that includes coronary artery disease, peripheral artery disease, cerebrovascular disease, and aortic atherosclerotic disease. In the United States, ASCVD affects approximately 1 in 10 people; represents a high risk for future major adverse cardiovascular events (MACE), such as heart attacks and strokes; and is the leading cause of death. ${ }^{1,2}$

Heterozygous familial hypercholesterolemia (HeFH) and other conditions that cause very high levels of lowdensity lipoprotein cholesterol (LDL-C) are associated with a high risk of premature ASCVD and MACE. ${ }^{3}$ Although $\mathrm{HeFH}$ is relatively common-affecting approximately 1 in 250 people in the United States-several studies have reported that the condition is still underdiagnosed and undertreated, particularly among women, Blacks, and Asians. ${ }^{4-6}$

LDL-C reduction is mainly achieved by high dose or maximally tolerated statin therapy. ${ }^{7,8}$ For patients who continue to have LDL-C levels at or above $70 \mathrm{mg} / \mathrm{dL}$, the addition of ezetimibe is recommended as second-line therapy, and if further $\mathrm{LDL}-\mathrm{C}$ reduction is necessary, a proprotein convertase subtilisin/kexin type 9 (PCSK9) inhibitor is recommended. ${ }^{9}$

A new option for lowering cholesterol is bempedoic acid (combined with ezetimibe [Nexlizet] or without ezetimibe [Nexletol], Esperion Therapeutics, Inc.), which was approved by the US Food and Drug Administration (FDA) in February 2020. Bempedoic acid is an inhibitor of adenosine triphosphate (ATP) citrate lyase that lowers LDL-C by reducing cholesterol synthesis upstream of HMG Co-A reductase (statins) and upregulating LDL receptors. ${ }^{10}$ Another new lipid-lowering agent, inclisiran (Novartis), is currently under FDA review. Inclisiran is a double-stranded small interfering RNA agent that lowers cholesterol through targeting and inhibiting hepatic PCSK9 synthesis. ${ }^{11}$

The Institute for Clinical and Economic Review (ICER) conducted a systematic literature review and cost-effectiveness analysis to evaluate the health and economic outcomes of bempedoic acid and inclisiran. In this report, we present the summary of our findings and highlight the policy discussion with key stakeholders held at a public

\author{
Author affiliations \\ Foluso Agboola, MBBS, MPH; Avery \\ McKenna; and Steven D Pearson, MD, \\ MSc, Institute for Clinical and Economic \\ Review, Boston, MA. Grace A Lin, MD, \\ MAS, Division of General Internal \\ Medicine and Philip R. Lee Institute \\ for Health Policy Studies, University of \\ California, San Francisco. Dhruv S Kazi, \\ MD, MSc, MS, Smith Center for Outcomes \\ Research in Cardiology, Beth Israel \\ Deaconess Medical Center and Harvard \\ Medical School, Boston, MA. \\ AUTHOR CORRESPONDENCE: \\ Foluso Agboola, fagboola@icer.org \\ J Manag Care Spec Pharm \\ 2021;27(7):961-66 \\ Copyright $@ 2021$, Academy of Managed \\ Care Pharmacy. All rights reserved.
}

meeting of the Midwest Comparative Effectiveness Public Advisory Council on February 5, 2021. The detailed report is available at https://icer. org/wp-content/uploads/2020/10/ ICER_High-Cholesterol_FinalEvidence-Report_030221.pdf. 


\section{Summary of Findings}

\section{CLINICAL EFFECTIVENESS}

We conducted pairwise meta-analyses on each drug separately. We did not attempt to compare these treatments with each other because of key differences across trials in patient characteristics and trial design. Additionally, we did not pursue a quantitative indirect comparison of inclisiran with PCSK9 inhibitors because trials evaluating the impact of inclisiran on cardiovascular outcomes have not been completed.

Bempedoic Acid. We identified 5 pivotal phase 3 randomized clinical trials (RCTs) of bempedoic acid with or without ezetimibe. ${ }^{10,12-15}$ Four studies examined bempedoic acid vs placebo, including 2 RCTs in patients with ASCVD or HeFH who required further LDL-C lowering despite being on maximally tolerated statin therapy ${ }^{10,14}$ and 2 RCTs in patients with ASCVD, HeFH, or hypercholesterolemia who were deemed to be statin intolerant, defined as the inability to tolerate trials of at least 2 statins..$^{12,15}$ The fifth RCT examined the combination pill bempedoic acid/ezetimibe vs bempedoic acid alone, ezetimibe alone, and placebo in patients with ASCVD, HeFH, or with multiple risk factors for cardiovascular disease while on maximally tolerated statins. ${ }^{13}$

Meta-analyses of the 5 RCTs $(\mathrm{N}=3,924)$ showed that bempedoic acid provided an overall $19.5 \%$ decrease in LDL-C after 12 weeks of treatment $\left(\mathrm{I}^{2}=69 \%, \mathrm{P}<0.01\right) \mathrm{com}^{-}$ pared with placebo. ${ }^{10,12-15}$ In patients with statin intolerance $(\mathrm{N}=614)$, a larger LDL-C reduction was observed with bempedoic acid compared with placebo (24.6\% vs $17.7 \%$; test for subgroup difference, $\mathrm{P}=0.05)^{12,15}$ Bempedoic acid also improved other lipid parameters, including significant reductions vs placebo in total cholesterol, non-high-density lipoprotein (HDL) cholesterol, apolipoprotein B, and highsensitivity C-reactive protein. Two of the RCTs presented data on cardiovascular events and mortality at 52 weeks. ${ }^{10,14}$ Meta-analyses of these studies for these endpoints found relative risks above 1 for all-cause mortality (rate ratio $[\mathrm{RR}]=2.25 ; 95 \% \mathrm{CI}=0.76-6.67)$ and cardiovascular mortality $(\mathrm{RR}=1.52 ; 95 \% \mathrm{CI}=0.41-5.70)$, but these studies were not powered to examine these endpoints, and numbers of patients with these outcomes were very small. ${ }^{10,14}$ Larger ongoing clinical outcome studies will assess these outcomes to evaluate fully how the reduction in LDL-C with bempedoic acid translates into patient-centered outcomes.

Data on adverse events (AEs) from existing trials show that more AEs were associated with bempedoic acid than placebo (24.1\% vs $20.3 \%, P=0.01)^{10,12,14,15}$ AEs that occurred with more frequency in the bempedoic acid group than the placebo group were muscle-related events (eg, pain in extremity, muscle spasms, and tendon rupture); hyperuricemia; gout; elevated liver enzymes (ALTand AST); and changes in renal laboratory parameters (eg, glomerular filtration rate and blood creatinine level).

Inclisiran. We identified three phase 3 RCTs of inclisiran vs placebo, 2 of which were conducted in patients with established ASCVD or ASCVD risk equivalent ${ }^{16}$ and 1 in patients with HeFH. ${ }^{11}$ All patients were on maximally tolerated lipidlowering therapy.

Meta-analyses of the 3 RCTs $(N=3,660)$ showed that inclisiran therapy decreased LDL-C levels by $51 \%$ from baseline (mean difference $=-50.5,95 \% \mathrm{CI}=-45.5$ to -55.5 ) compared with placebo after 77 weeks of treatment. ${ }^{11,16} \mathrm{~A}$ similar level of LDL-C reduction was observed in the study conducted exclusively in patients with $\mathrm{HeFH}(\mathrm{N}=482) .{ }^{11}$ Inclisiran also improved other lipid parameters compared with placebo, including an increase in HDL cholesterol and reductions in PCSK9, total cholesterol, non-HDL cholesterol, apolipoprotein B, and lipoprotein(a). Clinical outcome studies of inclisiran are also ongoing. Meta-analysis of prespecified exploratory cardiovascular endpoints reported in the phase 3 trials showed a lower rate of the composite cardiovascular outcome (cardiovascular mortality, cardiac arrest, non-fatal myocardial infarction, or stroke) with inclisiran compared with placebo $(\mathrm{RR}=0.76 ; 95 \% \mathrm{CI}=0.60-$ 0.96). ${ }^{11,16}$ The rates of cardiovascular death and all-cause mortality were similar between treatment arms.

Overall, a similar incidence of AEs, serious AEs, and discontinuation were observed between treatment groups. The most common $\mathrm{AE}$ with inclisiran was injection site reaction, which occurred in $5.4 \%$ of patients vs $0.8 \%$ in the placebo group. ${ }^{11,16}$

\section{LIMITATIONS OF CLINICAL EFFECTIVENESS}

Efficacy data on bempedoic acid and inclisiran are limited to short-term LDL reduction; long-term effect on clinical outcomes has yet to be established for either drug. Bempedoic acid blocks cholesterol synthesis upstream of statins, but it remains to be seen whether the degree of LDL-C lowering observed will translate into a similar degree of reduction in MACE rates as statin drugs. Additionally, bempedoic acid's safety profile raises important questions about whether the increased risk of hyperuricemia and gout, as well as a risk of tendon rupture, seen in the RCTs will be important real-world problems. For inclisiran, although the degree of LDL-C lowering appears to be in the same general range as found for PCSK9 inhibitors, whose mechanism of action lies along the same biochemical pathway, there remains uncertainty whether this will translate into a reduction in MACE rates that are more comparable to those seen with PCSK9 

for the Base Case

\begin{tabular}{|c|c|c|c|c|}
\hline $\begin{array}{c}\text { Treatment } \\
\text { (compared with statin + ezetimibe) }\end{array}$ & $\begin{array}{c}\text { Cost per } \\
\text { QALY gained, \$ }\end{array}$ & $\begin{array}{l}\text { Cost per } \\
\text { evLYG, \$ }\end{array}$ & $\begin{array}{l}\text { Cost per } \\
\text { LY gained, } \$\end{array}$ & $\begin{array}{l}\text { Cost per } \\
\text { MACE averted, \$ }\end{array}$ \\
\hline Inclisiran ${ }^{a}+$ statin + ezetimibe & $\$ 157,000$ & $\$ 142,000$ & $\$ 147,000$ & $\$ 451,000$ \\
\hline Bempedoic acid + ezetimibe + statin & $\$ 186,000$ & $\$ 168,000$ & $\$ 175,000$ & $\$ 535,000$ \\
\hline
\end{tabular}

${ }^{a}$ We used a hypothetical annual placeholder price of $\$ 5,644$ based on equivalent pricing to PCSK9 inhibitor drugs from the Federal Supply Schedule as of September 1, 2020, and assuming 2 doses per year. Initial treatment year requires 3 doses.

evLYG = equal value per life-years gained; $L Y=$ life-year; $M A C E=$ major adverse cardiovascular events; $Q A L Y=$ quality-adjusted life-year.

inhibitors or with statins. Finally, the trials of bempedoic acid and inclisiran did not include many patients from minority populations, who are disproportionately affected by ASCVD, and trials of bempedoic acid included very few patients with $\mathrm{HeFH}$, raising concerns about the generalizability of the findings.

\section{LONG-TERM COST-EFFECTIVENESS}

We evaluated the cost-effectiveness of bempedoic acid and inclisiran in patients with established ASCVD from a US health care sector perspective using a de novo statetransition Markov decision-analytic model. Our analyses compared each treatment plus ezetimibe and maximally tolerated statin therapy vs ezetimibe and maximally tolerated statin therapy only. The choice to model both treatments against statins plus ezetimibe stands in contrast to realworld data suggesting use of ezetimibe at less than $10 \%$ among patients who require further LDL-C lowering after statins. However, ezetimibe is now fully endorsed by clinical guidelines, and it has demonstrated mortality benefits.

Bempedoic acid is a more expensive oral alternative, and now that it is available, it is likely that more payers will require ezetimibe trials before approving insurance coverage for bempedoic acid or for PCSK9 inhibitors and inclisiran. Moreover, we believe that the true incremental value of these 2 new agents, and their associated valuebased price calculations, should be framed against the clinical and economic outcomes of statins plus ezetimibe.

For bempedoic acid, we chose to evaluate the costeffectiveness of its use in combination with ezetimibe (bempedoic acid/ezetimibe), since it is priced the same as bempedoic acid alone and so would be expected to dominate bempedoic acid in any economic evaluation. Separate evaluations included subgroups of patients with $\mathrm{HeFH}$, those intolerant to statins, and "high-risk" patients who have had acute coronary syndrome in the past year. The model used a lifetime time horizon, and costs and outcomes were discounted at $3 \%$ per year.
In the model, the relative effects of bempedoic acid/ ezetimibe and of inclisiran on LDL-C were "mapped" into a population representative of patients with ASCVD. Population characteristics were estimated from the National Health and Nutrition Examination Survey of US adults aged 35 years and older who have previous ASCVD and an LDL-C level of at least $70 \mathrm{mg} / \mathrm{dL}$ on statin therapy. ${ }^{17}$ The key input for the effectiveness of each drug was the percentage of reduction in LDL-C achieved at the primary endpoint among individuals receiving the therapy based on the meta-analyses of key clinical trials previously described. The LDL-C reduction observed with each drug was translated into reduction in MACE based on previous evidence for statin therapy, a key assumption given the absence of outcomes data. ${ }^{18}$ Full details on ICER's cost-effectiveness analysis and model are available on ICER's website at https://icer.org/ wp-content/uploads/2020/10/ICER_High-Cholesterol_ Final-Evidence-Report_030221.pdf.

Model results found that bempedoic acid/ezetimibe would produce modest improvements in clinical outcomes but that, at current estimated prices net of rebates and other concessions, the drug is unlikely to achieve commonly cited cost-effectiveness thresholds of $\$ 100,000-\$ 150,000$ per quality-adjusted life-year (QALY) or per equal value of life-years gained (evLYG; Table 1). For inclisiran, at a placeholder price of $\$ 5,644$ per year, set equivalent to the current average annual price for PCKSK9 inhibitor drugs, inclisiran approaches a cost-effectiveness threshold of \$150,000 per QALY and falls slightly below \$150,000 per evLYG (Table 1). Both treatments had better cost-effectiveness in higher risk subgroups, such as patients who are statin intolerant or who have HeFH.

\section{LIMITATIONS OF THE COST-EFFECTIVENESS MODEL}

As previously noted, clinical outcome studies on bempedoic acid/ezetimibe and inclisiran are ongoing. Thus, the LDL-C reductions observed with each drug in the short-term 


\section{TABLE 2 Votes on Contextual Considerations and Potential Other Benefits or Disadvantages for Any New} Effective Treatment for the Secondary Prevention of ASCVD

\begin{tabular}{|c|c|c|c|c|c|}
\hline & $\begin{array}{l}\text { Very low } \\
\text { priority }\end{array}$ & $\begin{array}{l}\text { Low } \\
\text { priority }\end{array}$ & $\begin{array}{l}\text { Average } \\
\text { priority }\end{array}$ & $\begin{array}{l}\text { High } \\
\text { priority }\end{array}$ & $\begin{array}{l}\text { Very high } \\
\text { priority }\end{array}$ \\
\hline $\begin{array}{l}\text { Acuity of need for treatment of individual patients based } \\
\text { on the severity of the condition being treated }\end{array}$ & 0 & 5 & 7 & 2 & 0 \\
\hline $\begin{array}{l}\text { Magnitude of the lifetime impact on individual patients } \\
\text { of the condition being treated }\end{array}$ & 0 & 2 & 5 & 5 & 2 \\
\hline
\end{tabular}

trials were translated into MACE reduction in our model based on previous evidence for statin therapy. If outcomes trials demonstrate that these relationships are substantially different, it will change the results of our analyses. Additionally, our economic evaluation assumes that statinintolerant patients are on no statin therapy, although real-world data suggest that many patients with statinassociated side effects can tolerate statins at low doses. Therefore, our findings would overestimate the clinical and economic benefit of lipid lowering if extrapolated to all patients with statin-associated side effects in real-world settings. Finally, our model did not examine primary prevention populations, which are at lower baseline risk for MACE than patients with established ASCVD.

\section{Policy Discussion}

The Midwest Comparative Effectiveness Public Advisory Council (CEPAC) is one of the independent appraisal committees convened by ICER to engage in the public deliberation of the evidence on clinical and cost-effectiveness of health care interventions. The Midwest CEPAC is composed of medical evidence experts, including practicing clinicians, methodologists, and leaders in patient engagement and advocacy. Their deliberation includes input from clinical experts and patient representatives specific to the condition under review and formal comments from manufacturers and the public. A policy roundtable concludes each meeting during which representatives from insurers and manufacturers join clinical experts and patient representatives to discuss how best to apply the findings of the evidence to clinical practice, insurance coverage, and pricing negotiations.

The ICER report on treatments for HeFH and secondary prevention of ASCVD was the subject of a Midwest CEPAC meeting on February 5, 2021. Following the discussion, the CEPAC members deliberated on key questions raised by ICER's report. The results of their votes on the clinical evidence are as follows:

1. The panel voted 9-5 that the clinical evidence was not adequate to demonstrate greater net health benefit of adding bempedoic acid to usual care in all patients with established ASCVD and/or HeFH when compared with usual care alone. The rationale given by panel members focused on the uncertainty resulting from a known potential for side effects, while LDL-C lowering was only modest and clinical outcomes trials were pending. However, in patients who have statin-associated side effects (statin-intolerant), the panel voted 12-2 that the evidence is adequate to demonstrate a net health benefit compared with usual care alone. Similarly, the panel voted 11-3 that the evidence was adequate to demonstrate a net health benefit of adding bempedoic acid to usual care for patients with $\mathrm{HeFH}$.

2. The panel voted 14-0 that there was adequate evidence to demonstrate a superior net health benefit of adding inclisiran to usual care compared with usual care alone in patients with established ASCVD and/or HeFH.

The Midwest CEPAC panel also voted on "contextual considerations" and "potential other benefits or disadvantages" as part of a process intended to signal to policymakers whether there are important considerations when making judgments about long-term value for money not adequately captured in analyses of clinical and/or cost-effectiveness. The results of these votes are shown in Tables 2 and 3 . They highlight several factors beyond the results of costeffectiveness modeling that the CEPAC panel felt were particularly important for judgments of overall long-term value for money.

The culminating vote of the CEPAC panel, intended to reflect its integration of the relevant elements of the value assessment framework, was on the "long-term value for money." A majority (13/14) of the panel members voted that the long-term value for money of combination pill bempedoic acid and ezetimibe plus usual care is low compared 


\section{TABLE 3 Votes on Contextual Considerations and Potential Other Benefits or Disadvantages for Bempedoic Acid Plus Usual Care vs Usual Care}

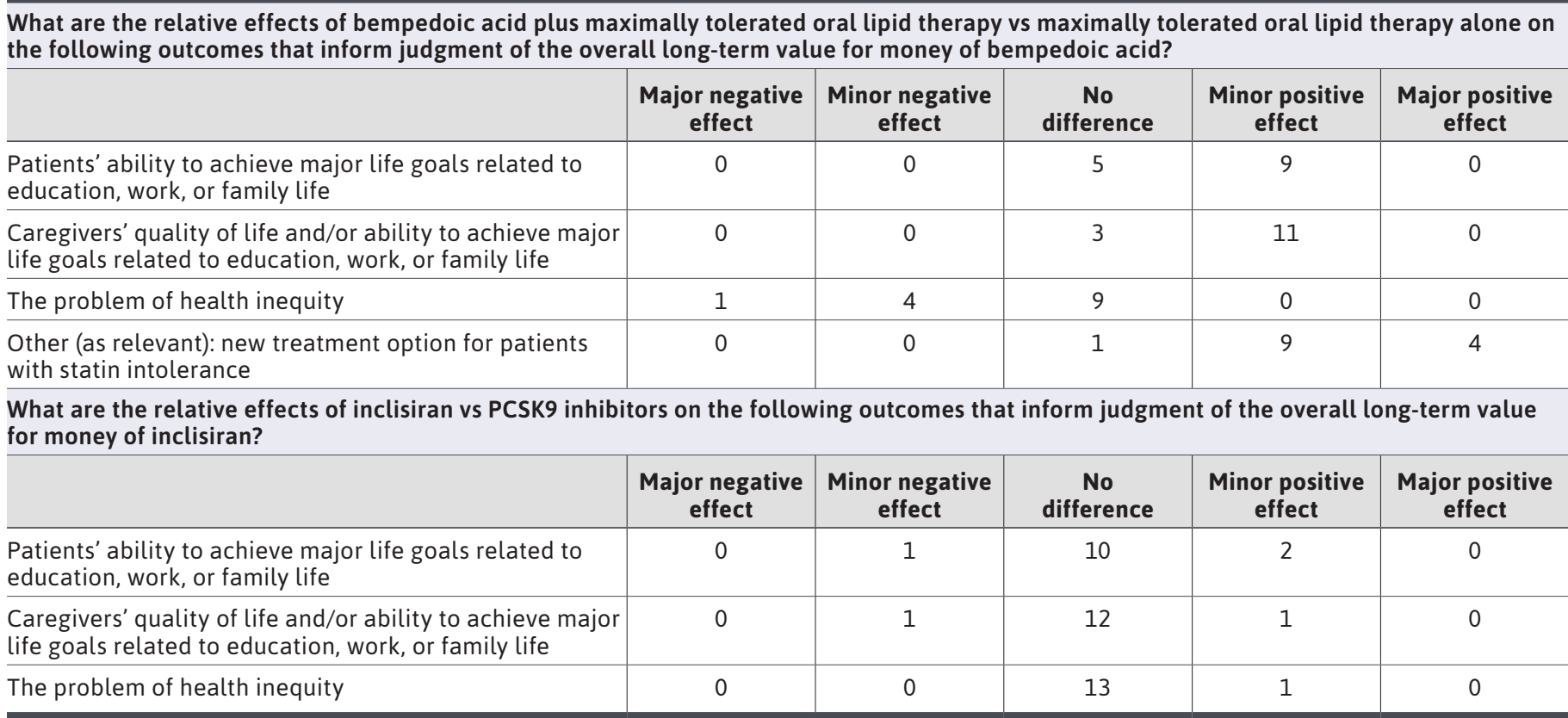

with usual care alone in all patients with established ASCVD and/or HeFH, a vote dominated by the uncertainty regarding clinical benefit and the base case cost-effectiveness above \$150,000 per QALY. At the current assumed price of inclisiran, a majority (10/14) of the panel judged that inclisiran represents a low long-term value for money in all patients with established ASCVD and/or $\mathrm{HeFH}$ when compared with usual care alone, a vote also colored by the lack of outcomes data and an estimated cost-effectiveness at the upper boundary of traditional cost-effectiveness thresholds.

The policy roundtable discussion explored how best to translate the evidence and additional considerations into clinical practice, pricing, and insurance coverage policies. The full set of policy recommendations can be found in the final evidence report on the ICER website: https:// icer.org/wp-content/uploads/2020/10/ICER HighCholesterol Final-Evidence-Report 030221.pdf. Several key policy recommendations follow:

- All stakeholders should ensure that the introduction of new therapies for high cholesterol do not exacerbate existing health inequities and should strive to decrease inequity in the health care system by decreasing cost and access barriers for patients to access effective therapies.
- All stakeholders should help increase awareness about the diagnosis and treatment of high cholesterol and address the underdiagnosis and HeFH.

- Payers should develop consistent prior authorization criteria for lipid-lowering drugs and ensure that the documentary burden and other administrative elements of prior authorization do not create an unreasonable burden on clinicians and patients.

- Payers should work with clinical experts and patient groups to develop consistent criteria and procedures for demonstrating drug intolerance due to statin associated side effects.

- Payers should ensure that coverage criteria reflect the status of higher-risk subpopulations for whom therapies may be both more clinically effective and cost-effective.

- For step therapy:

o Payers may wish to consider step therapy with ezetimibe before bempedoic acid or inclisiran, since some patients may reach their LDL-C goal with the combination of statin and ezetimibe, both of which are generic drugs and have been shown to improve cardiovascular outcomes.

o But if patients are unlikely to reach their LDL-C goals with the addition of ezetimibe alone (eg, $\geq 25 \%$ above their LDL-C goal), payers should allow coverage for the 
combination pill of bempedoic acid with ezetimibe or inclisiran/PCSK9 inhibitor without requiring a first step through ezetimibe.

\section{DISCLOSURES}

Funding for this summary was contributed by Arnold Ventures, California Health Care Foundation, The Donaghue Foundation, Harvard Pilgrim Health Care, and Kaiser Foundation Health Plan to the Institute for Clinical and Economic Review (ICER), an independent organization that evaluates the evidence on the value of health care interventions. ICER's annual policy summit is supported by dues from AbbVie, Aetna, America's Health Insurance Plans, Anthem, Alnylam, AstraZeneca, Biogen, Blue Shield of CA, BoehringerIngelheim, Cambia Health Services, CVS, Editas, Evolve Pharmacy, Express Scripts, Genentech/Roche, GlaxoSmithKline, Harvard Pilgrim, Health Care Service Corporation, HealthFirst, Health Partners, Humana, Johnson \& Johnson (Janssen), Kaiser Permanente, LEO Pharma, Mallinckrodt, Merck, Novartis, National Pharmaceutical Council, Pfizer, Premera, Prime Therapeutics, Regeneron, Sanofi, Spark Therapeutics, uniQure, and United Healthcare.

Agboola, McKenna, and Pearson are employed by ICER. Lin and Kazi received funding from ICER for work on this report.

\section{ACKNOWLEDGMENTS}

The authors thank Rick Chapman, Katherine Fazioli, Maggie O'Grady, and Monica Frederick for their contributions to this report.

\section{REFERENCES}

1. Xu J, Murphy SL, Kockanek KD, Arias E. Mortality in the United States, 2018. NCHS Data Brief. 2020;(355):1-8.

2. Benjamin EJ, Blaha MJ, Chiuve SE, et al. Heart disease and stroke statistics-2017 update: a report from the American Heart Association. Circulation. 2017;135(10):e146-e603.

3. Singh A, Gupta A, Collins BL, et al. Familial hypercholesterolemia among young adults with myocardial infarction. J Am Coll Cardiol. 2019;73(19):2439-50.
4. Perez de Isla L, Alonso R, Watts GF, et al. Attainment of LDL-cholesterol treatment goals in patients with familial hypercholesterolemia: 5-year SAFEHEART Registry follow-up. J Am Coll Cardiol. 2016;67(11):1278-85.

5. Zafrir B, Shapira C, Lavie G, Halon DA, Flugelman MY. Identification and characterization of severe familial hypercholesterolemia in patients presenting for cardiac catheterization. J Clin Lipidol. 2016;10(6):1338-43.

6. Amrock SM, Duell PB, Knickelbine T, et al. Health disparities among adult patients with a phenotypic diagnosis of familial hypercholesterolemia in the CASCADE-FH ${ }^{\mathrm{TM}}$ patient registry. Atherosclerosis. 2017;267:19-26.

7. Fihn SD, Gardin JM, Abrams J, et al. 2012 ACCF/AHA/ACP/AATS/PCNA/SCAI/STS guideline for the diagnosis and management of patients with stable ischemic heart disease: a report of the American College of Cardiology Foundation/ American Heart Association Task Force on Practice Guidelines, and the American College of Physicians, American Association for Thoracic Surgery, Preventive Cardiovascular Nurses Association, Society for Cardiovascular Angiography and Interventions, and Society of Thoracic Surgeons. J Am Coll Cardiol. 2012;60(24):e44-e164.

8. Grundy SM, Stone NJ, Bailey AL, et al. 2018 AHA/ACC/AACVPR/AAPA/ ABC/ACPM/ADA/AGS/APhA/ASPC/ NLA/PCNA guideline on the management of blood cholesterol: a report of the American College of Cardiology/ American Heart Association Task Force on Clinical Practice Guidelines. Circulation. 2019;139(25):e1082-e1143.

9. Lloyd-Jones DM, Morris PB, Ballantyne CM, et al. 2016 ACC expert consensus decision pathway on the role of non-statin therapies for ldl-cholesterol lowering in the management of atherosclerotic cardiovascular disease risk: a report of the American College of Cardiology Task Force on Clinical Expert Consensus Documents. J Am Coll Cardiol. 2016;68(1):92-125.
10. Ray KK, Bays HE, Catapano AL, et al. Safety and efficacy of bempedoic acid to reduce LDL cholesterol. N Eng J Med. 2019;380(11):1022-32.

11. Raal FJ, Kallend D, Ray KK, et al. Inclisiran for the treatment of heterozygous familial hypercholesterolemia. N Eng J Med. 2020;382(16):1520-30.

12. Ballantyne CM, Banach M, Mancini GBJ, et al. Efficacy and safety of bempedoic acid added to ezetimibe in statin-intolerant patients with hypercholesterolemia: a randomized, placebo-controlled study. Atherosclerosis. 2018;277:195-203.

13. Ballantyne CM, Laufs U, Ray KK, et al. Bempedoic acid plus ezetimibe fixed-dose combination in patients with hypercholesterolemia and high CVD risk treated with maximally tolerated statin therapy. Eur J Prev Cardiol. 2020;27(6):593-603.

14. Goldberg AC, Leiter LA, Stroes ESG, et al. Effect of bempedoic acid vs placebo added to maximally tolerated statins on low-density lipoprotein cholesterol in patients at high risk for cardiovascular disease: the CLEAR Wisdom Randomized Clinical Trial. JAMA. 2019;322(18):1780-88.

15. Laufs U, Banach M, Mancini GBJ, et al. Efficacy and safety of bempedoic acid in patients with hypercholesterolemia and statin intolerance. J Am Heart Assoc. 2019;8(7):e011662.

16. Ray KK, Wright RS, Kallend D, et al. Two phase 3 trials of inclisiran in patients with elevated LDL cholesterol. N Eng J Med. 2020;382(16):1507-19.

17. Centers for Disease Control and Prevention. NHANES survey methods and analytic guidelines. 2020. Updated April 23, 2021. Accessed June 4, 3021. https:// wwwn.cdc.gov/nchs/nhanes/analyticguidelines.aspx\#analytic-guidelines\%20 (2020).

18. Cholesterol Treatment Trialists Collaboration; Baigent C, Blackwell L, Emberson J. Efficacy and safety of more intensive lowering of LDL cholesterol: a meta-analysis of data from 170,000 participants in 26 randomised trials. Lancet. 2010;376(9753):1670-81. 